\section{CONTINUOUS SYSTEMS}

\subsection{INTRODUCTION}

System modelling through simulation has been traditionally divided into two areas; discrete event simulation and continuous process simulation. However, many systems are a mixture of discrete and continuous processes, and artifically forcing them into the discrete or continuous mould will generally result in an inadequate model.

Several attempts have been made to combine these two modelling approaches to handle such systems of interacting discrete and continuous processes. Apart from certain difficulties of implementation on digital computers, these languages perform 'off-line' simulations with very limited interaction capabilities.

A very desirable feature for simulating discrete event or combined discrete-continuous systems which include human operators is to have good on-line operator-computer interaction and visual feedback of the progress of the simulation to the operator. This feature is of great help not only in gaining good insight into the problem but also in having the model operations verified by people who understand the physical system well, but who are not necessarily familiar with the actual modelling process and coding involved in the simulation.

In this paper, the simulation of interacting discrete and continuous systems on a hybrid computer is discussed. A hybrid computer was chosen mainly for the relative ease with which display facilities could be employed, and for the on-line interaction capability available in a hybrid computer environment.

With the analog portion handling all the continuous parts of the simulation, increased problem size and complexity in this part requires more analog components, but does not increase simulation time. The digital computer handles the discrete event simulation portion as well as control of the analog computer. Since only one event is executed at any given time, a method of overlay may be employed in a mini-computer if a disk operating system is available. Thus, core size does not affect the size

\author{
P. W. U. Graefe, I. K. Nenonen ${ }^{1}$ \\ and K.A. Strobele ${ }^{2}$ \\ 1 National Research Council of Canada \\ 2 Hatch Associates Ltd.
}

of problems which can be simulated. In the studies carried out by the authors, an EAI 640 digital computer with $16 \mathrm{~K}$ of memory easily handled problems whose total core requirements were 60 to $100 \mathrm{~K}$ words.

\subsection{IMPLEMENTATION OF COMBINED DISCRETE CONTINUOUS SIMULATION ON A HYBRID. COMPUTER}

Conceptually, it is easiest to think of the discrete part of the combined simulation as an event oriented process, as used in SIMSCRIPT or GASP, for instance. These discrete event simulation facilities work essentially as follows.

After initialization, a file containing events ordered chronologically by their time of execution is accessed, and the first time event removed. Simulation time is set to the time of execution of this event and the event is executed. This may result in the execution of other 'non-time' events and/or scheduling. of other 'time' or 'non-time' events. Following this, the next time event is removed from the time file, simulation time is advanced instantaneously to this new time, and this next time event is executed. This sequence of action continues until some end of simulation condition is encountered.

This mode of operation is quite easily altered to a combined discrete-continuous simulation. The basic change is to allow continuous advance of time for the solution of the continuous submodels between the discrete time instances when events are being executed. This requires an analog timing circuit on which time targets can be set by the digital computer, and which can notify the digital computer when this time target has been reached. The difference in event times between the present time event and that of the event just removed from the time file is used as a time target and fed to a comparator via a digital-to-analog converter. The comparator compares this time target with the output of a timing integrator. Upon reaching a time target the output of this integrator is reset to zero and the digital computer is notified of the fact via a sense line. At any time during the simulation the output of the timing integrator can be read through 
HYBRID SIMULATION $\ldots$ Continued

an analog-to-digital converter giving the elapsed time in minutes since the occurrence of the last time event. Thus the simulation time can be established at any time desired.

Simulation submodels may be implemented either in a purely digital form using the discrete event structure, or they may be hybrid models using analog components to solve dynamics with the digital computer doing the necessary bookkeeping whenever analog targets have been reached. Examples of hybrid submodels drawn from a scheduling study in a copper smelter (Ref. 1) are:

1. Display of simulation time. Here the simulation time in hours and minutes can be read by the model operator from digital voltmeter displays.

2. Material handling cranes. These cranes operate on the same track and cause considerable interference. Crane position information is constantly available through servo-driven crane models.

3. Copper converter: The differential equations of the reaction are solved on the analog computer and

"the solutions can be monitored continuously via strip chart recorders.

Display units (Ref. 2) containing many multicoloured lights, servo-driven cranes, digital voltmeters etc. are easily interfaced with the hybrid computer to provide an excellent feedback of information for an on-line operator.

\section{BIBLIOGRAPHY}

1. Graefe, U., Nenonen, L.K., Strobele, K., "Copper Smelter Scheduling Study Using a Hybrid Computer", IFAC Symposium on Automatic Control in Mining, Mineral and Metal Processing, Sydney, Australia, August 13-17, 1973, pp. 219-223.

2. Graefe, U., Nenonen, L.K., Strobele, K., "Simulation of Industrial Operations with Improved Man/Model Interface", Proceedings of the Canadian Conference on Automatic Control, University of New Brunswick, September 24-25, 1973, pp. 8.1-1, to 8.1-16. 